\title{
Constraining SN Ia Models Using X-ray Spectra of Clusters of Galaxies
}

\author{
Renato A. Dupke ${ }^{1}$ and Raymond E. White III $^{2,3}$ \\ ${ }^{1}$ Dept. of Astronomy, University of Michigan, Ann Arbor, MI 48109-1090 \\ ${ }^{2}$ Code 662 NASA/GSFC Greenbelt, MD 20771 \\ ${ }^{3}$ Dept. of Physics 69 Astronomy, University of Alabama, Tuscaloosa, AL 35487
}

\begin{abstract}
We present constraints on theoretical models of Type Ia SNe using spatially resolved ASCA X-ray spectroscopy of four galaxy clusters: Abell 496, Abell 2199, Abell 3571 \& Perseus. All four clusters have central Fe abundance enhancements and an ensemble of abundance ratios are used to show that most of the Fe in the central regions of the clusters comes from SN Ia. At the center of each cluster, simultaneous analysis of spectra from all ASCA instruments shows that the $\mathrm{Ni}$ to $\mathrm{Fe}$ abundance ratio (normalized by the solar ratio) is $\approx 4$. We use the $\mathrm{Ni} / \mathrm{Fe}$ ratio as a discriminator between $\mathrm{SN}$ Ia models: the $\mathrm{Ni} / \mathrm{Fe}$ ratio of ejecta from the Convective Deflagration model W7 is consistent with the observations, while those of delayed detonation models are not consistent at the $90 \%$ confidence level.
\end{abstract}

\section{INTRODUCTION}

Type Ia SNe are thought to be generated by thermonuclear explosions of carbonoxygen white dwarfs undergoing accretion in stellar binary systems[1]. The nature of the progenitor binary systems, the masses of the white dwarfs and the explosion mechanism(s) (e.g. convective deflagration, delayed-detonation, etc.) for SN Ia are still open questions $[2,3]$. The explosion of a $\mathrm{Ch}$ mass $\mathrm{C}-\mathrm{O}$ white dwarf is thought to be initiated by carbon ignition at the center, followed by a subsonic nuclear flame (deflagration wave) propagating outwards. In the classical W7deflagration model [4], the propagation speed of the flame front is relatively high $(\approx 15-25 \%$ of the $c_{s}$ ), but remains subsonic. In delayed detonation models(WDD), the flame speed is initially much lower $\left(\approx 1-3 \% \mathrm{c}_{s}\right)$, but rises to become supersonic $[5,6]$.

The existence of heavy elements in the intracluster medium (ICM) indicates that part of the ICM is not primordial, i.e. was processed in stars and injected into the ICM. The inferred ICM Fe abundances are typically in the range 0.3-0.4 Solar. X-ray spectroscopy of the ICM has indicated a dominance of SN II $[7,8]$. However, theoretical uncertainties in the elemental yields from SN Ia \& II allow ASCA spectroscopy to also be interpreted as showing that as much as $50 \%$ of the Fe in clusters comes from SN Ia $[9,10,11]$ and may rise to $\approx 70 \%$ in the vicinity

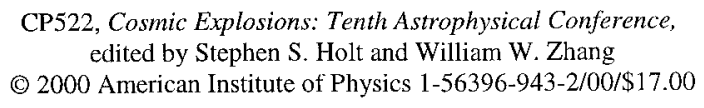


of its central cD galaxy $[12,13,14]$, which may be due to a secondary SN La-driven wind (post-protogalactic wind), partially suppressed in the vicinity of the $\mathrm{cD}$.

Despite the general agreement on the predicted elemental mass yields among different SN Ia models, W7 \& WDD models predict significantly different mass yields for some elements (e.g. Ni). Accurately determining the elemental yields from $\mathrm{SN}$ Ia is crucial to determining the relative contribution of different SNe types to the metal enrichment of galaxies and ICM. Thus, determining which enrichment mechanism(s) were most dominant in contaminating the ICM (e.g. protogalactic, ram pressure stripping, SN Ia-driven winds). Furthermore, by comparing the observed abundance ratio values to those predicted by different SN Ia models, we can constraint theoretical SN Ia models .

We analyze ASCA X-ray spectra of the central regions of 4 galaxy clusters: A496, A2199, A3571 \& Perseus. The central regions of these cluster are shown to be dominated by SN Ia ejecta $(\approx 65-80 \%)$. The abundance measurement uncertainties allow to discriminate between competing SN Ia models. We use an ensemble of different elemental abundance ratios to determine the SN Ia Fe mass fraction. This allows us to check for self-consistency when comparing different SN Ia models.

\section{SN Ia Fe MASS FRACTION \& DELAYED DETONATION MODELS}

Radial distributions of global abundances are shown in Fig. 1a. All clusters show mild, but significant, central abundance enhancements. The best-determined abundances in all four clusters are those of $\mathrm{Fe}, \mathrm{Si} \& \mathrm{Ni}$. O, Ne \& S abundances are not well constrained in Abell 3571. Ni abundances are about twice solar in all four clusters. To estimate the SN Ia/II mass fraction, we compare various observed abundance ratios to the theoretical predictions of specific models for SN Ia \& II. For SN Ia we initially adopt the updated W7 model of Nomoto et al. [15], while for SN II we use the calculations of Nomoto et al. [15,16], who adopt a Salpeter initial mass function over a SN II progenitor mass range of $10-50 \mathrm{M}_{\odot}$.

Dupke \& White [12] and Dupke \& Arnaud [14], using different theoretical models for SN II yields, found that the theoretical S yield would have to be reduced by a factor of 3 and that the theoretical Ne yields would have to be increased by a factor of 2.7 to be consistent with results for most other elemental ratios. Therefore, to be conservative, we avoid including abundance ratios involving these two elements in the calculation of SN Ia Fe mass fraction. We use only abundance ratios involving the better constrained (observationally and theoretically) abundances of $\mathrm{Fe}, \mathrm{Si}, \mathrm{Ni}$ $\& \mathrm{O}$. The SN Ia Fe mass fraction derived from abundance ratios involving the above elements is listed in Table 1 for A496 and Perseus, where weighted averages are denoted WA. Errors are propagated $90 \%$ confidence errors for Perseus and 1- $\sigma$ for A496. Dashed missing values indicate that the abundance ratio values is outside the boundaries between SN Ia \& II. The results for A3571 and A2199 are similar. 


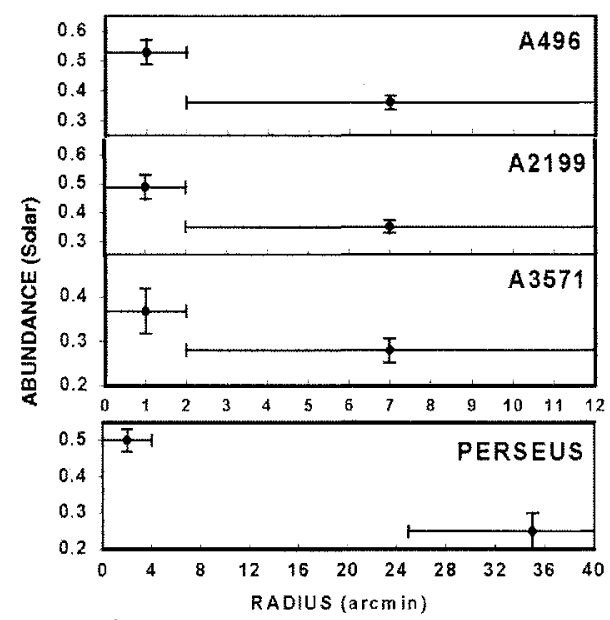

a)

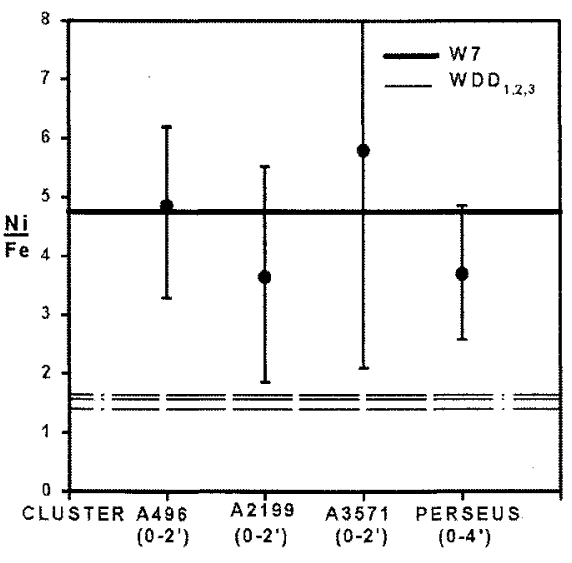

b)

FIGURE 1. (a) - Radial Abundance Distributions. The central bin of all four clusters are spatially similar $\left(\approx 100 \mathrm{~h}_{50}^{-1} \mathrm{kpc}\right.$ ). Errors are $90 \%$ confidence. $(\mathrm{b})$ - Ni/Fe Abundance Ratio for the Central Clusters Regions. Predictions for SN Ia enrichment based on the W7 (solid thick) and 3 different WDD (dotted thin) models. SN II models predict $\mathrm{Ni} / \mathrm{Fe} \approx 1.9$.

As seen in Table 1, a variety of abundance ratios provide mutually consistent estimates of the central SN Ia Fe mass fraction. This mutual consistency encourages us to test alternative theoretical models for SNe Ia. In particular, we compare the W7 model adopted above to delayed detonation models. We consider the delayed detonation models of Nomoto et al. [15], who calculate yields for a sequence of models distinguished by a variety of densities ahead of the deflagration front, which modulates the onset of detonation. The results are also shown in Table 1 . We find that the elemental yields of their models WDD1, WDD2 \& WDD3 do not provide the same consistency as found with model W7 in estimating the SN Ia/II fraction.

It can be seen that W7 model provides more satisfactory results in three different ways: 1) more abundance ratios fall within the theoretical bounds of SN Ia \& II, as they should, when W7 is adopted; 2) the dispersion in the average of the (remaining) individual SN Ia Fe mass fraction estimates tends to be smaller for the W7 model; 3) the mutual consistency of the SN Ia Fe mass fraction estimates is best for W7 model.

$\mathrm{Ni}$ is the most discriminatory element between WDD \& W7 (displaying a large range of variation of mass yields). The $\mathrm{Ni}$ abundance is reasonably well constrained in the X-ray spectra of clusters with ASCA. Fe has the most precisely determined abundance. Therefore, we use the $\mathrm{Ni} / \mathrm{Fe}$ ratio as a SN Ia model discriminator. Fig. $1 \mathrm{~b}$ compares the $\mathrm{Ni} / \mathrm{Fe}$ ratio observed in the four clusters analyzed in this work to the $\mathrm{Ni} / \mathrm{Fe}$ ratio predicted by the $\mathrm{W} 7$ deflagration model, as well as from the three delayed detonation models described at Nomoto et al. [15]. The observed Ni/Fe 


\begin{tabular}{|c|c|c|c|c|c|}
\hline \multirow[t]{2}{*}{ Cluster } & \multirow{2}{*}{$\begin{array}{l}\text { Element } \\
\text { Patio }\end{array}$} & \multicolumn{4}{|c|}{ SN Ia Iron Mass Fraction } \\
\hline & & W7 & WDDI & WDD2 & WDIS3 \\
\hline A.496 & $O / F$ & $0.77^{+0.13}$ & $0.82+0.11$ & $0.77^{+0.12}$ & $0.74^{+0.12}$ \\
\hline$" r$ & $\mathrm{Si} / \mathrm{Fe}$ & $0.65+0.07$ & $\geq 0.96$ & $0.79+0.08$ & $0.65+0.09$ \\
\hline er & $\mathrm{Ni} / \mathrm{Fe}$ & $1.00_{-0.27}^{+0.00}$ & - & - & $\longrightarrow$ \\
\hline$\prime \prime$ & $\mathrm{O} / \mathrm{Si}$ & $0.86+0.18$ & $0.72 \pm 0.17$ & $0.77 \pm 0.75$ & $0.81 \pm 0.12$ \\
\hline$\prime \prime$ & $S i / N i$ & $0.74+8.07$ & - & - & - \\
\hline$\Rightarrow$ & $V A A$ & $0.73 \pm 0.05$ & $0.82+0.11$ & $0.78 \pm 0.07$ & $0.68+0.07$ \\
\hline Perseus & $\mathrm{O} / \mathrm{Fe}$ & $0.67+0.24$ & $0.73+0.29$ & $0.67+0.24$ & $0.63+0.26$ \\
\hline$m$ & $\mathrm{Si} / \mathrm{Fe}$ & $0.70+0.10$ & $\geq 0.98$ & $0.84 \pm 0.11$ & $0.69 \pm 0.11$ \\
\hline$\prime r$ & $\mathrm{Ni} / \mathrm{Fe}$ & $0.66_{-0.35}^{+0.34}$ & - & - & - \\
\hline 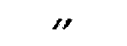 & $\mathrm{O} / \mathrm{Si}$ & $0.59+0.38$ & $0.37+0.55$ & $0.43+0.50$ & $0.50_{-0.45}^{+0.45}$ \\
\hline 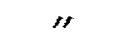 & $\mathrm{Si} / \mathrm{N} \mathrm{i}$ & $0.69+0.17$ & - & $\ldots$ & $\geq 0.92$ \\
\hline$" \prime$ & $V A$ & $0.69 \pm 0.07$ & $0.66+0.21$ & $0.79+0.11$ & $0.67 \pm 0.10$ \\
\hline
\end{tabular}

ratios are inconsistent with any of the delayed detonation models at better than $90 \%$ confidence. The observed Ni/Fe ratio is fully consistent with the W7 model.

ACKNOWLEDGMENTS. This work was partially supported by the NSF and the State of Alabama through EPSCoR grant EHR-9108761. REW also acknowledges partial support from NASA grant NAG 5-2574 and a National Research Council Senior Research Associateship at NASA GSFC. RAD acknowledges partial support from NASA grant NAG 5-3247. This research made use of the HEASARC ASCA database and NED.

\section{REFERENCES}

1. Hoyle, F., \& Fowler, W. A. ApJ 132, 565 (1960).

2. Branch, D. ARAA 36, 17 (1998).

3. Nomoto, K., Iwamoto, K., \& Kishimoto, N. SCIENCE 276, 1378 (1997).

4. Nomoto, K., Thielemann, F.-K., \& Yokoi, K., ApJ 286, 644 (1984).

5. Arnett, W. D. \& Livne, E. ApJ 315, 565 (1994a).

6. Arnett, W. D. \& Livne, E. ApJ 330, 565 (1994b).

7. Mushotzky, R. F., \& Loewenstein, M. ApJL 481, L63 (1997).

8. Mushotzky, R. F., et al. PASJ 49, 1 (1997).

9. Ishimaru, Y., \& Arimoto, N. PASJ 50, 187 (1998).

10. Fukazawa, Y., et al. ApJ 132, 565 (1960).

11. Nagataki, S., \& Sato, K., ApJ 504, 629 (1998).

12. Dupke, R. A., \& White, R. E. III $A p J$ astro-ph/9902112, submitted (1999).

13. Dupke, R. A., \& White, R. E. III $A p J$ astro-ph/9907343, inpress (2000).

14. Dupke, R. A., \& Arnaud, K. A. ApJ submitted (1999).

15. Nomoto, K., et al. Nuclear Physics A A621, 467c (1997b).

16. Nomoto, K., et al. Nuclear Physics A A616, 79 (1997a). 